\title{
Opacity modulation for interactive volume rendering of medical images
}

\author{
Alberto Signoroni, Francesca Manerba, Riccardo Leonardi \\ Signals and Communications Lab. - DEA - University of Brescia, Italy \\ via Branze, 38 - I25123 Brescia, Italy Tel.+39030 3715432 Fax.+39030 380014
}

\begin{abstract}
Interactivity is a main requirement for 3D visualization of medical images in a variety of clinical applications. The good matching between segmentation and rendering techniques is essential to design easy to use interactive systems which assist the physicians in dynamically creating and manipulating "diagnostically relevant" images from the volumetric data sets. In this work we consider the above problem within an original interactive visualization paradigm. By this paradigm we want to highlight the twofold clinical requirement of a) detecting and visualizing the structures of diagnostic interest (SoDI's) and b) adding to the 3D scene some other structures to create a meaningful visual context. Being the opacity modulation of the different structures a crucial point, we propose an opacity management which reflects the paradigm ideas and operates by means of a twofold indexed look-up table (2iLUT). The 2iLUT consists of a combination of attribute based and object based opacity management and is here designed and experimented in order to combine the time interaction benefits of an indexed opacity setting with the effective handling of the above classification and visualization clinical requirements.
\end{abstract}

Keywords: Clinical applications of 3D imaging, segmentation of 3D data sets, interactive visualization, objectbased analysis, morphology, volume rendering, opacity transfer functions.

\section{INTRODUCTION}

In the last decade there has been a great diffusion of 3D medical image visualization tools because they provide physicians with volumetric informations about anatomical and pathological structures, morphology or functional mappings, hardly obtainable using the very slice by slice analysis. ${ }^{1}$ Thus, $3 \mathrm{D}$ visualization can be used to support physicians in diagnoses, preoperative planning, postoperative validations and other clinical applications ${ }^{2,3}$; however, obtaining diagnostic relevant pictures from 3D data sets on a single case basis constitutes a challenge both for image analysis and interaction issues, because of the unavoidable 3D to 2D lossy projection which entails (even with the possibility to interactively manipulating the projection perspective) a drastic reduction of the visual informative content hidden in the volumetric data set. Actually there is a trade-off between such losses and the value added by the augmented representation space. A good object or material classification (which usually involves a segmentation) and a correct management of the related color and opacity are key points in order to find a satisfactory solution to the above compromise. In the biomedical field, due to the high complexity of the low to high level visual features of the measured physical data (e.g. a MR or CT scan), and due to the semantic nature of the diagnostic task, the skill of the physician are essential, and its role critical, in order to define (segment) and classify the Structures of Diagnostic Interest (SoDI). This is especially true when pathological (abnormal) situations necessarily involves a subtle and specialized reasoning not affordable even for the most sophisticated unsupervised information analysis tools. The objective to obtain diagnostically relevant $3 \mathrm{D}$ views $^{*}$, as visual representation of the salient (from a diagnostic point of view) 3D features of the considered clinical data, is actually wide-ranging, but presents some highlights which can be summarized by 1) the necessity to focalize the attention on the SoDI's and 2) the need to consider them in their meaningful context. The first point corresponds to a semantic need of a 3D view representing the 3D SoDI in an accurate and reliable way,

Authors e-mail: \{signoron,manerba,leon\}@ing.unibs.it

${ }^{*}$ With $3 \mathrm{D}$ view we refer to a projected $2 \mathrm{D}$ image (or a couple of stereoscopic images) of a $3 \mathrm{D}$ scene which in turns is defined on a $3 \mathrm{D}$ data set. 
the second one has more or less the same characteristics, but in the customary case of limited computational and interaction resources, we observed that the accuracy of the context visual information becomes less critical, while another issue comes out: contrary to the static nature of the SoDI information, the context would be better considered a dynamic set of objects with the possibility to modulate the opacity of different objects in an independent way. This can be accomplished by having the possibility to "pick-up" some of the structures and let it intervene into the scene. From a diagnostic point of view this should be essential for example to evaluate (by inspection or by quantitative tools) the exact position of the SoDI with respect to some reference structure or markers. Another very common necessity is the possibility to evaluate the impact and the interactions of a pathological SoDI with respect to its surrounding structures; this would give the physician a better diagnostic sight and a 3D perception of the field of intervention with a consequential better planning of the correlated strategies and implications. A panoramic view of clinical application of $3 \mathrm{D}$ rendring of biomedical volumes can be found for example in the paper of Shaidi et al. ${ }^{2}$ and in the Bankman's handbook. ${ }^{3}$

In our work we first propose a paradigmatic approach to the problem of producing diagnostic relevant images in an interactive framework (Sec.2), then we concentrate on a solution, based on direct volume rendering (DVR) that could be adopted to produce such images by guaranteeing a good degree of interactivity and satisfactory results, even in the case of limited graphics resources. That solution is based on the possibility to define a so called twofold-indexed look-up-table (2iLUT) in order to manage different type of objects in a dynamic way by their introduction into the scene and the modulation of their opacity (Sec.3). The objects are differentiated in SoDI's and structures of contextual interest (SoCI's). In Sec.4 we propose and examine an automatic analysis tool to segment the whole volume into labeled objects in order to let the 3D scene composition easy and intuitive. At this point (Sec.5) the 2iLUT idea is described in detail as well as the related implementation choices and the allocation policies. Finally, we propose some interactive visualization results (Sec.6) and give some remarks and our conclusions (Sec.7).

\section{INTERACTIVE VOLUME RENDERING PARADIGM}

To explain the core of our reasoning and to justify the implementation strategies, we first propose a general paradigm to which the subsequent technological solutions should be inspired. This paradigm concerns a general view of the representation and visualization problem in a diagnostic scenario and keeps into account the key role of a physician who interacts with the computational tools in order to obtain the desired results. With this paradigm we emphasize two conceptually different moments, which in turns constitutes two mutually interlaced interaction loop. At first, the physician is concentrated to isolate, with a high desired level of accuracy, the SoDI's and this will be called interactive segmentation. In a second moment the need to have a global view of the diagnostic context is taken into account, as stated in the previous section, and this is accomplished by an interactive visualization. The interactive (or alternatively guided) segmentation and visualization expressions stand for automatic algorithms which accept and wait for some key parameters coming (transduced) from an external word, i.e. an application environment where the user needs to operate at a semantic level. On the other hand the user perceives the computational tools as actuators of complex and time consuming information representation tasks but at the same time should have the possibility to guide that computation in the right direction, in an effective way, toward the desired result. An interesting overview of the interactive segmentation today scenario can be found in the work of Olabarriaga and Smeulders. ${ }^{4}$

In Fig:2 we show a scheme of the proposed information representation paradigm. The starting point is the Original Volume data OV. At this point we foresee a bifurcation toward two interactive tasks: one directed to segment the SoDI's and the other directed to build the diagnostically relevant images. The scope of the Interactive (IS) is to extract from (OV) a volume formed by one or more anatomic or pathological structures of diagnostic interest. After this process it is possible to visualize (for example by an integrated view of slices and volumetric rendering) the segmented volume $(\mathbf{S g V})$ and to evaluate if it satisfy the radiologist's needs. If it doesn't, the segmented volume can be used as a starting point for a refinement step, or the whole segmentation process repeated, using different settings. Once successfully obtained, a final segmented volume (FV) can be generated. At this point, if there is the exigency to better contextualize the FV result with respect to the entire volumetric data set, the second interactive task, represented in Fig.1 by the interactive visualization module (IV), take places. Ideally, this activity is conceived to exploit the results of a "global" segmentation 


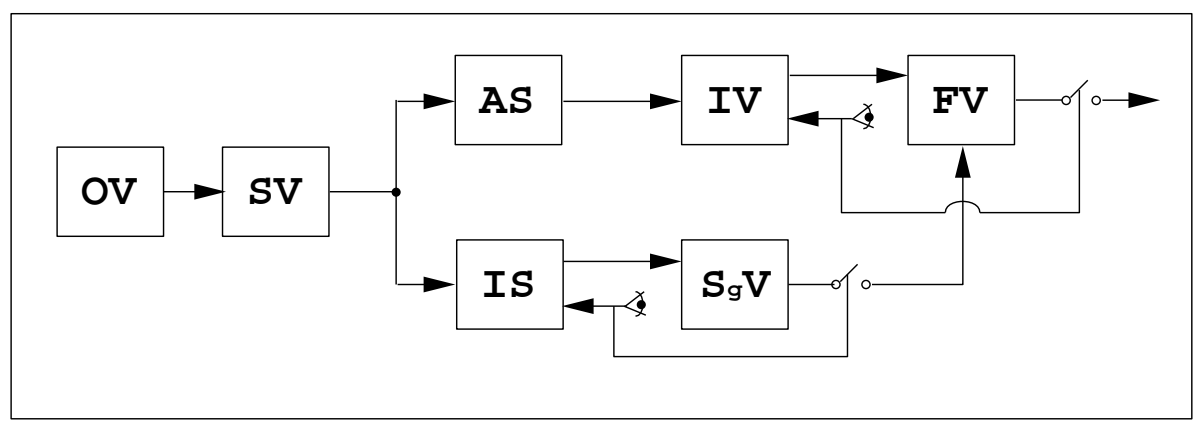

Figure 1: The data representation and visualization paradigm

of the volume. However, a whole volume segmentation is usually out of the scope of a supervised approach, especially for medical volumes analyzed in a clinical environment, because of the great number and heterogeneity of the structures, the related anatomical classification effort and an unlikely employment of an high skill, to accomplish such an annoying, error prone and time expensive task. However, being the classification necessary to a meaningful rendering we foresee an automatic segmentation AS, which should partition the volume in coherent objects which in turns can be "encouraged" to come on the scene, with a certain degree of opacity. A set of one or more of these objects should constitute a SoCI which contribute to create a context for the SoDI's. This actually entails an object based interaction, where 3D objects could be recalled by the physician by a direct indication of them on the $2 \mathrm{D}$ slices and with an initial opacity assignment that can be further modulated by the visual feedback. This way the SoDI's should be still visible but, at the same time, the physician has the possibility to evaluate their spatial and functional relation with respect to the surroundings structures (SoCI's). Following the spirit of this paradigm we design a solution to allow the physicians to accomplish the critical tasks of creating diagnostically relevant 3D views of the composed 3D scene. Such technological solution guarantees an affordable interaction effort and provide a high-level semantic guiding of some task-oriented analysis and visualization tools which in turns are be based on some non-critical basic hypothesis. Such hypothesis allow to exploit the available computational and graphics resources. In particular we concentrate on the interactive visualization branch of our paradigm, while the IS adopted to produce the results presented in this work is described in Benini et al.. ${ }^{5}$

\section{OPACITY MODULATION AND OBJECT BASED INTERACTION}

We concentrate on a visual representation of 3D scenes which is based on a direct volume rendering (DVR) of the voxels. ${ }^{6-8}$ In its nature, DVR presents some advantages with respect to the widespread surface rendering in terms of degree of realism and because of the wide-ranging of achievable image features, but it presents some additional computational costs and until now suffered from a lesser dedicated hardware supply. A DVR which guarantees good degree of interactivity and satisfactory visual results in critical environments (such as the medical imaging one) is a still open field of investigation. Key issues are the assignment of a suitable opacity to the voxels or to the objects of a volume and the possibility to interactively modulate that opacity. The opacity value may be linked on a voxel basis to the color or gray level value, either by an implicit association or more commonly by the addition of an opacity value $\alpha$ to the voxel luminance or color (RGB $\alpha$ approach). The opacity value can also be derived by other measurable attributes (typically a morphological gradient level, in order to highlight discontinuities and make the uniform regions completely transparent) or more generally by a function of a set of attributes. Another possibility is to assign the opacity on a classification basis, where the classification may be related to different materials or to different objects. The object classification is generated by a segmentation process which could be automatic, guided (interactive) or manual. The way the $3 \mathrm{D}$ view is created depends on the particular DVR technique but, even if it is out of the scope of this paper to give a volume rendering state-of-the-art, a basic DVR computing distinction can be made between two different approaches: the direct mode and the indexed mode. 
Direct mode: the 3D views are directly calculated from the RGB $\alpha$ voxel values. The maximum degree of freedom is provided in terms of color-opacity combinations, but at each modification (e.g. opacity modulation) the original volume rewriting and the volume rendering pipeline re-computation are required.

Indexed mode: a Look-Up Table (LUT) is used to establish a relation between some volume features and the opacity voxel value. The index $i$ of the LUT could be either implicit or explicit. In the first case the stored voxel value is the color value in the second case the memory contains an RGB $i$ volume, where $i$ comes from a classification or segmentation pre-processing. A so called opacity transfer function (OTF) lies in the LUT and is read only during the last stage of the rendering pipeline, the compositing stage. An opacity modulation consists in rewriting the OTF (without modifying the stored volume data) followed by the compositing re-computation.

The indexed mode is limited by the existence of a transfer function between a voxel feature (or a features combination) and the opacity value assigned to the voxel, but usually allows for a more intuitive and immediate opacity modulation, with reduced computational costs. On the other hand, associating a single opacity value to each voxel, without using LUT's, limits real-time interaction with the data. As a consequence the indexed mode is better suited for interactive applications. Generally, the LUT length could be limited too. This limit usually derive from software or hardware restrictions.

Now we return to the problem of rendering a scene with a SoDI and a dynamic set of SoCI's. We would like to find an opacity modulation solution that benefits from the LUT performance without suffering from the combinatorial limitations due to the feature based OTF which prevents from an object based opacity modulation. A first solution could be using (if possible) a longer or more than one LUT. This would give the possibility to implement more than one OTF by exploiting an a-priori volume segmentation and a relative (static) volume partition in different classes, each one with its own OTF. However this is not a true object based approach and doesn't allow, as requested in our case, to put in or take off a SoCI from the scene in an interactive way. Another possibility is to use what we call a twofold indexed look-up table, 2iLUT. This is a subdivision of the LUT into two regions which are indexed in different ways. One region is dedicated to the SoDI's and works as a normal LUT (feature based indexing), the other region is indexed by the labels which corresponds to different SoCI's with their own opacity value (object based indexing). In Sec.5 a detailed description of the 2iLUT opacity management is given. Before doing that, we briefly describe the criteria and the solution we adopted to perform the whole volume object segmentation.

\section{UNSUPERVISED WHOLE VOLUME SEGMENTATION}

In Sec.2 an automatic segmentation, AS (see Fig.1) of the whole volume has been justified to the extent that the segmented structures serve to create a visual context for the SoDI's. Interacting with the result of an AS, a physician may have the possibility to quickly recall some segmented structures which should actually constitute a meaningful set of visualized SoCI's. Apart from the problem to select an effective segmentation engine, other main problems to deal with are the over-segmentation and under-segmentation control. Biomedical CT or MR volumes are noisy data; noise generates uncertainty and, as a consequence, an over-segmentation tendency. On the other hand the exigency to detect objects with a certain morphological coherence should generate undersegmentation problems. By keeping in mind that the physician can quickly select the desired SoCI (e.g. by directly pointing them on the $2 \mathrm{D}$ slices) we can find a certain clearance in our trade-off by tolerating some slight over-segmentation. For example we allow a kidney to be built by 2 or 3 segmented objects. This way we do not burden the "pick-up" interaction and have a margin against the under-segmentation.

The implementation is based on a 3D morphological AS preceded by a volume simplification based on a 3D extension $^{9}$ of the connected operators analysis. ${ }^{10}$ We experimented that a volume simplification presents several benefits for the subsequent segmentation in that: 1) it operates a contour preserving noise removal reducing the partitioning uncertainty, 2) it diminishes the number of segmented regions, by removing the irrelevant one, according to a certain a-priori defined filtering criterion (for example a volume measure) and 3) allows to reduce 
the over-segmentation problems ${ }^{\dagger}$.

On the simplified volume we calculate a thresholded 3D morphological gradient followed by a 3D region growing expansion in order to find the boundary of the different objects and assign to each one a different label. To better exploit the transparency visual effect of the selected SoCI, we associate a non-zero opacity only to the voxel which are on the boundary of the objects. Due to the gradient thresholding, the complex nature of objects, the residual noise and other factors (which depends on several image and imaging instrument features), the 3D gradient, which acts as a barrier for the region growing, usually presents small but fatal holes. Even a good threshold setting could not completely resolve this problem. To gain robustness to this problem we perform a region growing with structuring elements which extent is sufficiently large (usually $2^{3}$ to $4^{3}$ voxel cubes) to prevent the object boundary trespassing.

In Fig.2 we consider the case of an abdominal CT volumetric data set. The scope here is to select, from the segmented objects, the spine and the pelvis which are the SoCI's. The figure shows (a) the whole data set represented by a DVR with a linear OTF, (b) the quick visual interaction on a slice to indicate, by the marking points, the spine, and (c) the same slice on the volume of the segmented objects contours. The interaction leads to the choice of only three objects (indicated in Fig.2(c) by the arrow) which will intervene into the 3D scene (see next section). Here the spine results to be connected to the pelvis and does not require additional markers to be selected.
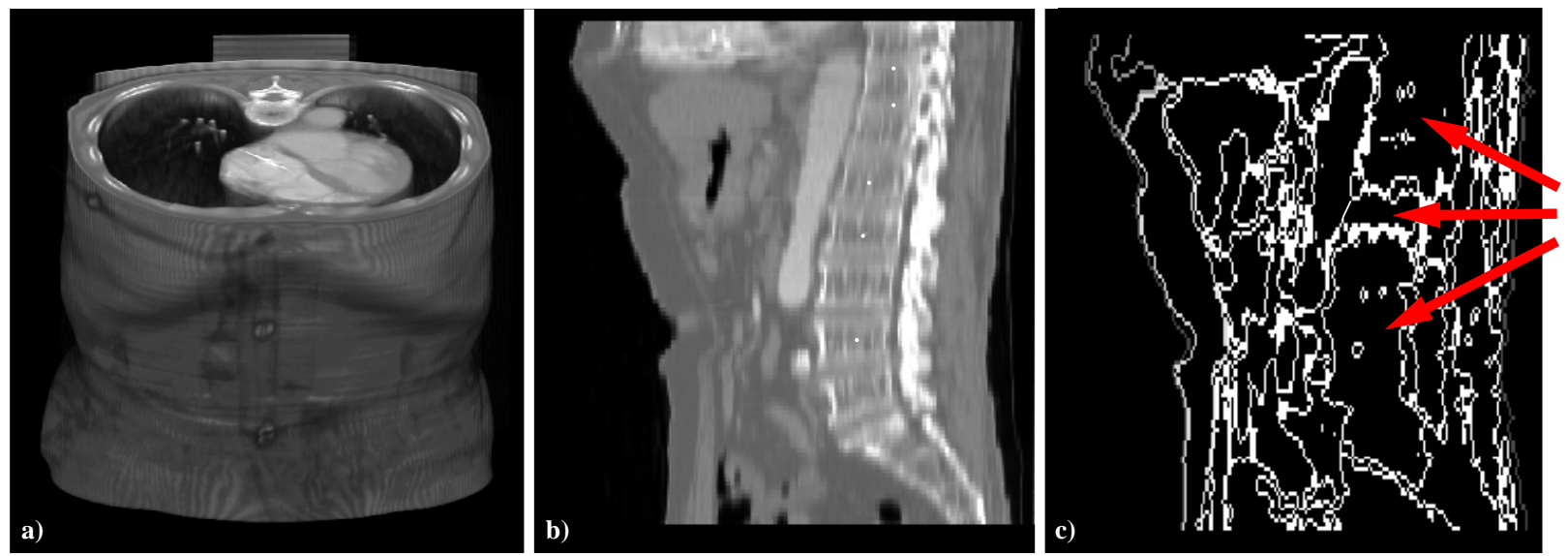

Figure 2. CT abdominal data set: (a) DVR with linear OTF. (b) a volume slice with the markers placed to which indicate the SoCI's; (c) the same slice with the sectioned $3 \mathrm{D}$ contours of the segmented objects; the arrows indicates the 3 objects which result to be selected by the markers.

\section{OBJECT BASED OPACITY MODULATION WITH 2ILUT}

The 2iLUT defined in Sec.3 requires a certain volume index management to be coupled with the paradigm presented in Sec.2. As in the case of feature based OTF, when the feature is not the luminance (or gray level), a volume of voxel attributes has to be created, in association to the data volume, for LUT indexing purpose. With the twofold indexing we are subjects to a 2iLUT entry partition problem. However, we observe that the number of luminance levels of a SoDI is usually only a fraction of the total. Thus, even in the case of a LUT with a number of entries limited to the number of gray levels, the above observation can be exploited in order to allocate the remaining LUT entry to contain the opacity value of the SoCI's. The Tab.1 shows the structure of the volume and the 2iLUT in the typical case of a single SoDI (the multiple SoDI case is an easy generalization). As volume data we consider, for each voxel $v=(x, y, z)$, two associated values: the gray level (or color) $g(v)$

\footnotetext{
${ }^{\dagger}$ As we will see in the next section, reducing the number of regions is important to simplify the management of the $2 \mathrm{iLUT}$ because it involves lesser "overbooking" problems.
} 


\begin{tabular}{|c|c|c|c|c|}
\hline Voxel, $v$ & Volume & Volume & 2iLUT & 2iLUT \\
\hline Description & Gray level, $g$ & Label, $l$ & Index, $i$ & Opacity, $o$ \\
\hline \hline $\begin{array}{c}\text { Segmented object } \\
\text { boundary }\end{array}$ & $g(v)$ & $\begin{array}{c}l(v,(\mathrm{AS})) \\
f=1\end{array}$ & $i=A(l)$ & $o=\operatorname{IV}(i)$ \\
\hline $\begin{array}{c}\text { Segmented object } \\
\text { interior }\end{array}$ & $g(v)$ & $\begin{array}{c}l(v,(\mathrm{AS})) \\
f=0\end{array}$ & 0 & 0 \\
\hline SoDI & $g(v)$ & $L$ & $i=I(g)$ & $o=\operatorname{OTF}(i)$ \\
\hline Others & $g(v)$ & 0 & 0 & 0 \\
\hline
\end{tabular}

Table 1: Structure of the volume and the 2iLUT.

and the voxel attribute $l$ which in our case is the label of an AS object, with a flag $f$ indicating whether the voxel is on the boundary or inside the object. The 2iLUT index $i$ is obtained observing the label $l$ and the flag $f$. When $v$ is on the boundary of the segmented object $l$ the 2iLUT index $i$ assumes a value which is a function of the label, $i=A(l)$, according to the underlying object allocation policy (see below). For the considered voxels the 2iLUT contains a value defined during the interactive visualization: $\operatorname{IV}(i)=0$ when the object is not selected, elsewhere $\operatorname{IV}(i)$ assumes a value decided by the physician. If $v$ belongs to the interior of an object (flag=0) the index is set to zero, and points to the zero opacity cell. For voxels belonging to the SoDI there is a reserved value $l=L$ which corresponds to a reserved region of the 2iLUT, for convenience the last part, which is indexed by a function of the gray level, $I(g)$. That function simply translates the gray level range of the SoDI: $I(g)=N-\lambda_{\max }+g$, with $\lambda_{\max } \leq g \leq \lambda_{\max }$ where $N$ is the 2iLUT dimension and $\lambda_{\min }$ and $\lambda_{\max }$ are the SoDI gray level extremes. That portion of 2iLUT contains an OTF to represent the SoDI with a good degree of realism. A typical choice is the linear $\operatorname{OTF}(g)=g / M$, where $M$ is the maximum gray-scale value and the opacity represented on the $[0,1]$ interval. The remaining unclassified voxels also have a 2 iLUT index equal to zero.

To clarify what happens we continue the example of the previous section. In the presented case of a volumetric CT data set the SoDI actually corresponds to an aorta tract while, as we said, the SoCI's are the spine and the pelvis. In Tab.2 a 2iLUT summary is presented for this case: the three selected objects are the only automatically segmented objects which opacity $\alpha$ is different form zero. Here the $A(l)$ association is simply $A(l)=l$. For the SoDI we indicates the $g$ extremes $\left(\lambda_{\min }\right)$ and $\left(\lambda_{\max }\right)$ and the corresponding index and opacity extremes. Fig.3 graphically represents the 2iLUT, while in Fig.4 we show four different DVR views of the 3D scene, calculated with a 2iLUT opacity modulation of the SoCI's.

\begin{tabular}{|l|l|c|c|}
\hline Object type & Description & Index, $i$ & Opacity, $o$ \\
\hline \hline SoCI 1 & spine & 35 & $\alpha$ \\
SoCI 2 & spine & 39 & $\alpha$ \\
SoCI 3 & spine and & 149 & $\alpha$ \\
& pelvis & & \\
\hline SoDI $\left(\lambda_{\min }\right)$ & aorta & 201 & 0.549 \\
SoDI $\left(\lambda_{\max }\right)$ & & 255 & 0.761 \\
\hline
\end{tabular}

Table 2: Example of 2iLUT assignments.

If the 2iLUT dimension is not limited then we can accommodate one or more OTF for the SoDI's and all the necessary entries corresponding to the automatically segmented object. If it is not the case sometimes some overbooking problems may arise. In fact it may happen that the SoDI OTF, which extent is $\lambda_{\max }-\lambda_{\min } 2 \mathrm{iLUT}$ entries, occupies a great portion of the 2iLUT, otherwise the number of segmented objects should surpass the LUT capacity. However, this is not a big issue because there are more than one strategy that can be combined to sort out this eventuality:

Object number reduction The gradient threshold could be slightly elevated to reduce the number of 


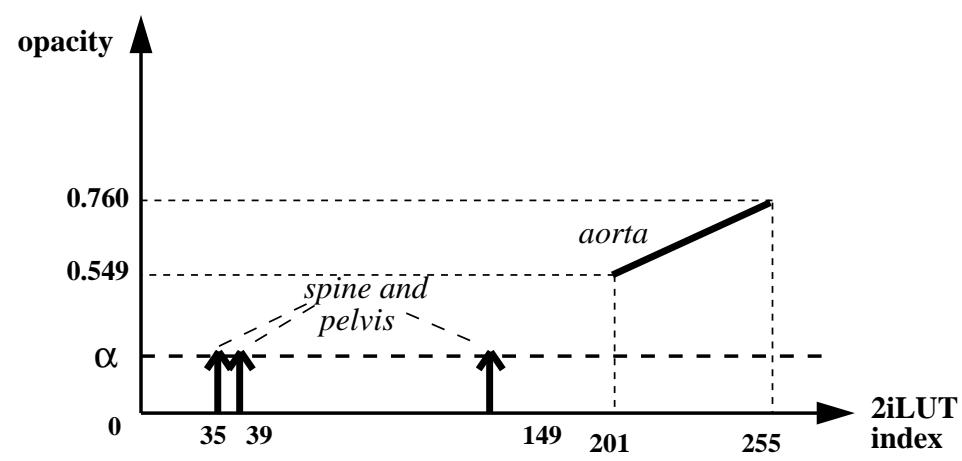

Figure 3: Graphic representation of the 2iLUT

segmented region, or other region merging strategies could be adopted. This implies a volume rewriting.

Object label prioritizing It is possible to create a prioritizing hierarchy by assigning the labels in an importance order, where importance is determined by a certain criterion, for example the dimension of the object or the SoCI to SoDI distance. According to the free 2iLUT entries, only the most important segmented structures are actually indexed.

IV decimation A merging of the segmented objects can be implemented without having to rewrite the volume by modifying the $A(l)$ function in order to assign the same index value to different $l$ values.

OTF decimation Usually it is also possible to decimate the OTF without impairing the visual result.

A right strategy prevents from a visual impairment or from the inability to obtain "diagnostically relevant" images.

\section{INTERACTIVE VISUALIZATION SYSTEM AND RESULTS}

In order to evaluate both functional and computational performance, we realized a software prototype using the OpenGL and OpenVolumizer 1.2 libraries $^{11}$ on a SGI O2 workstation (CPU MIPS R5000 200MHz with FPU, CRM Graphics 1280x1024 32+32 bit-planes). This system is quite surpassed and actually limits the number of LUT entries to 256 , however it is enough to guarantee good interactive visualization performance and to evaluate the potentiality of our framework. With the software it is also possible to perform the guided segmentation of the SoDI's. ${ }^{5}$ When both IS and AS are accomplished, the 3D volume rendering of the previously segmented SoDI's, and three slices of the volume taken along the three orthogonal axes are presented in the main window. The physician can move and zoom the 3D scene and navigate among the slices. Such interaction is fluid thanks to the grid subsampling during the motion. From the same window the physician can choose which structures he'd like to see in transparency near the SoDI's by picking up them from one or more slices and choose a suitable transparency value for each of them. No memory rewriting is required for these two operations which produce visual results in about $1 \mathrm{sec}$. This delay has been judged not objectionable by the physicians in that, due to the discrete time nature and the high semantic level of the interaction, only near real-time performance are required. We now present two examples of diagnostically relevant images produced with our system where the interaction was performed by a neuroradiologist.

Gray matter In Fig. 5 a comparison on a $256^{2} \times 141$ MR data set is proposed between the visualization of the gray matter alone (which is the SoDI) and the same structure with the upper part of the skullcap and the skin as SoCI's. The high degree of accuracy of the SoDI representation, which is relevant for diagnostic purposes, is preserved by modulating the opacity of the SoCI's which in turns are relevant for preoperative planning and/or postoperative validation. The SoDI has been obtained in a few minutes of guided segmentation, while 

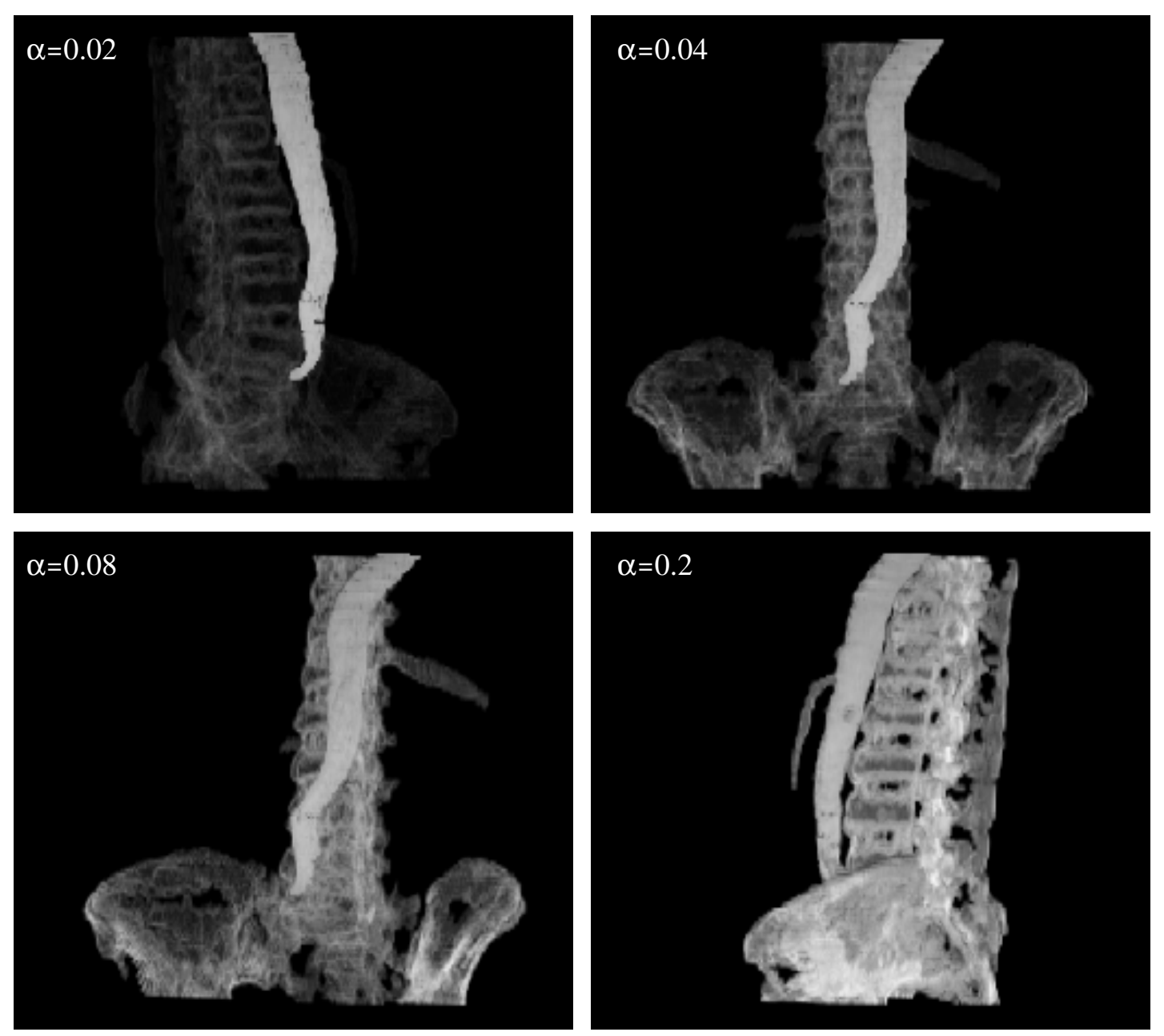

Figure 4. Interactive visualization example: the SoDI is the aorta and the SoCI's are the spine and the pelvis that are opacity modulated using the $2 \mathrm{iLUT}$.

the skullcap and the skin corresponds to two automatically segmented objects picked-up and put into the 3D scene by the neuroradiologist in a few seconds.

Intracerebral meningioma with cerebral cortex The second example presents the case of a volumetric MR study which reveal a brain meningioma. The lesion has been segmented and showed in Fig.6(a). However, for diagnostic purposes it is necessary to evaluate the impact and the interaction of the meningioma with the surrounding structures, in this case the brain and in particular the cerebral cortex. In fact the small appendix of the meningioma could be an important diagnostic element (called dural tail sign) to ascertain the benign nature of the tumor. Fig.6(b) and (c) show two 3D views which has been judged, in the terms stated before, diagnostically relevant, and allows a satisfying inspection of the $3 \mathrm{D}$ scene thanks to the opacity modulation. Now if there is the need to plan an intervention or to evaluate other aspects the bone and the skin could be added and this is presented in Fig.7 and Fig.8: the part (a) shows the detail of the markers (the white points) placed by the neuroradiologist and (b) the corresponding $3 \mathrm{D}$ views which appears to the screen. 

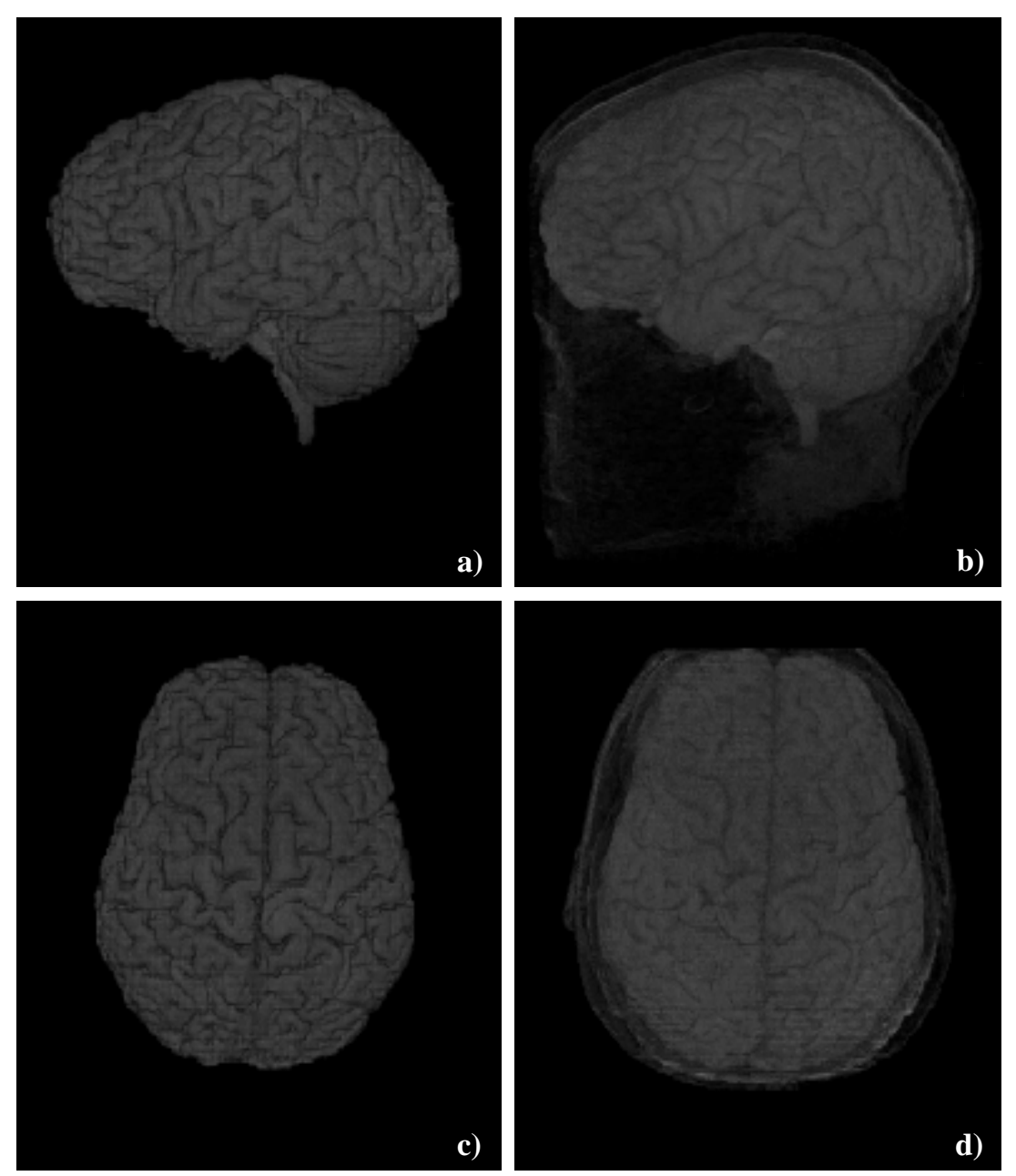

Figure 5. Two different views of the gray matter (a),(c) and the corresponding ones obtained with the addition of the skullcap and the skin with opacity $\alpha=0.04(\mathrm{~b}),(\mathrm{d})$.

\section{REMARKS AND CONCLUSIONS}

The paradigm, the solutions and the prototype presented in this work are aimed to decompose the problem of obtaining "diagnostic relevant" images, as 3D views of a volumetric scene, into different units. The units concerns different objectives, requirements, and interaction languages. In particular the interactive segmentation has the objective to obtain, with a high degree of accuracy, the SoDI which need to be represented with the best degree of realism, but also need the additional presence of some SoCI which, depending on the diagnostic context, allow to create the meaningful 3D scene. The SoDI are treated as solid objects whereas the SoCI are voided in order to better represent their surface with a user defined degree of opacity, and this entails some visualization requirements which are different for SoDI's and SoCI's. In order to manage those requirements in an effective way a twofold indexed LUT has been introduced. This solution extends the possibility of a classical attribute based LUT toward an object based opacity attribution. The 2iLUT management is based on the IS+AS voxel labeling and allows to quickly and effectively obtain meaningful 3D views, even on low-cost PC's and workstations. The working hypothesis of the interactive visualization paradigm allow in practice to guide the visualization toward results which presents the combinatorial power of the RGB $\alpha$ DVR without the need to rewrite the volume at each interaction. This bears benefits in terms of computational time and interactivity. The realized prototype implements the functionality of the interactive visualization paradigm and 

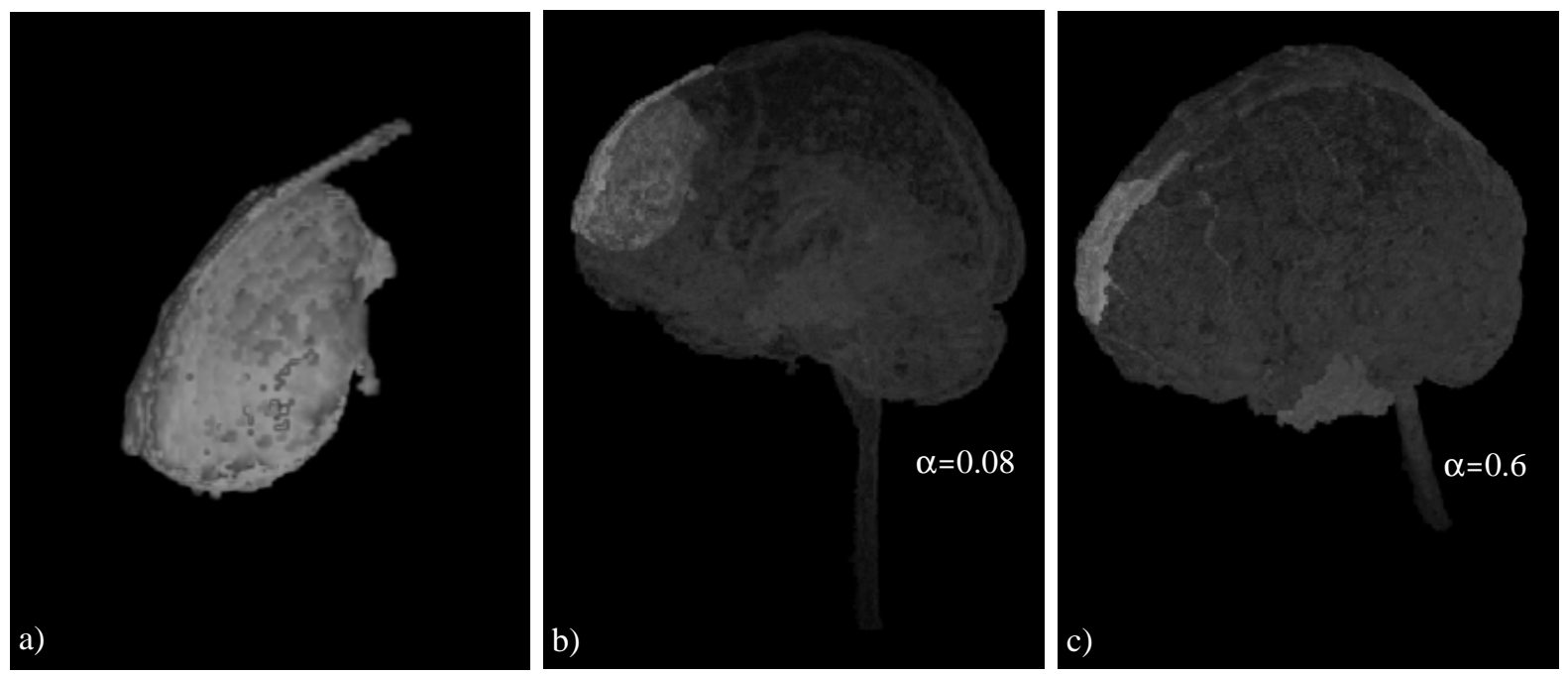

Figure 6: The brain meningioma alone (a) and with the semi-transparent (b) and near-opaque (c) cortex.
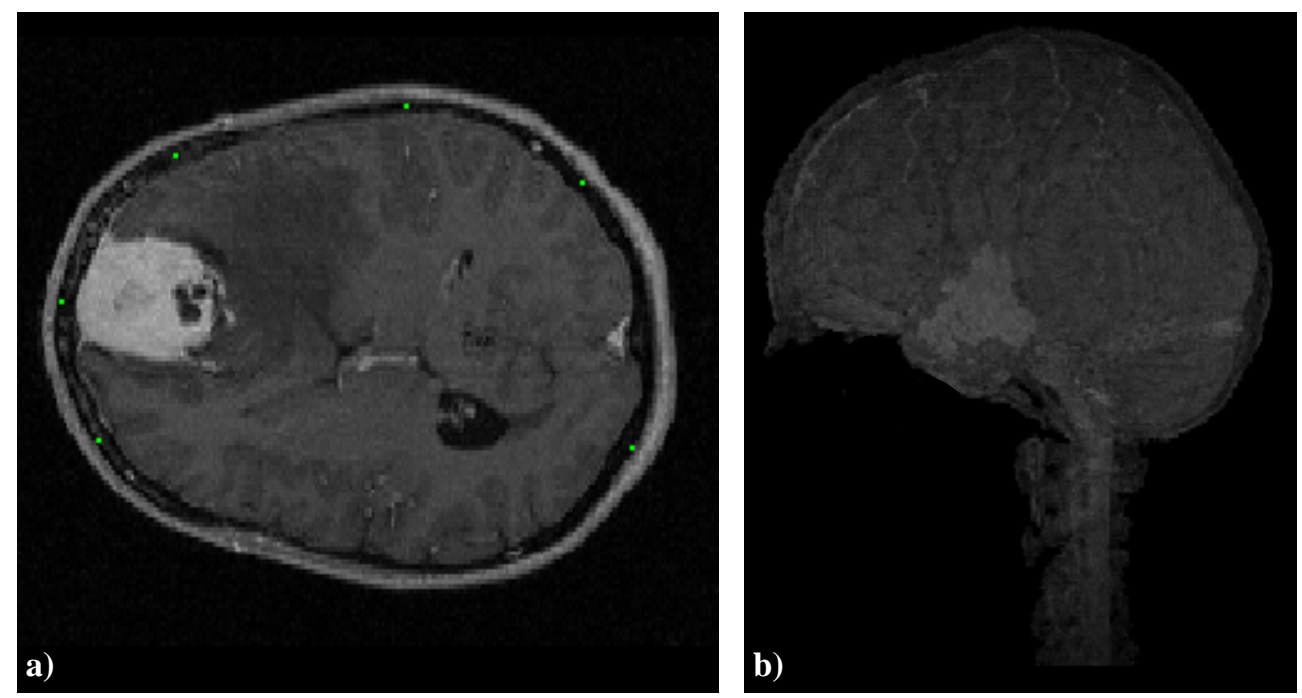

Figure 7: Skullcap addition: the point placement (a) and the visual result (b).

has demonstrated its potential to allow the physicians to obtain diagnostic relevant images in an effective way by interactively working with different kind of data sets in a variety of diagnostic situations.

\section{ACKNOWLEDGMENTS}

Prof. Roberto Gasparotti and Dr. Lorenzo Pinelli of the Neuroradiology division of the Spedali Civili and University of Brescia for their essential contribution to the experimental part of this work.

\section{REFERENCES}

1. J. S. Duncan and N. Ayache, "Medical image analysis: progress over two decades and the challenges ahead," IEEE Trans. on Pattern Analys. and Machine Intell. 22, pp. 85-106, Jan. 2000. 

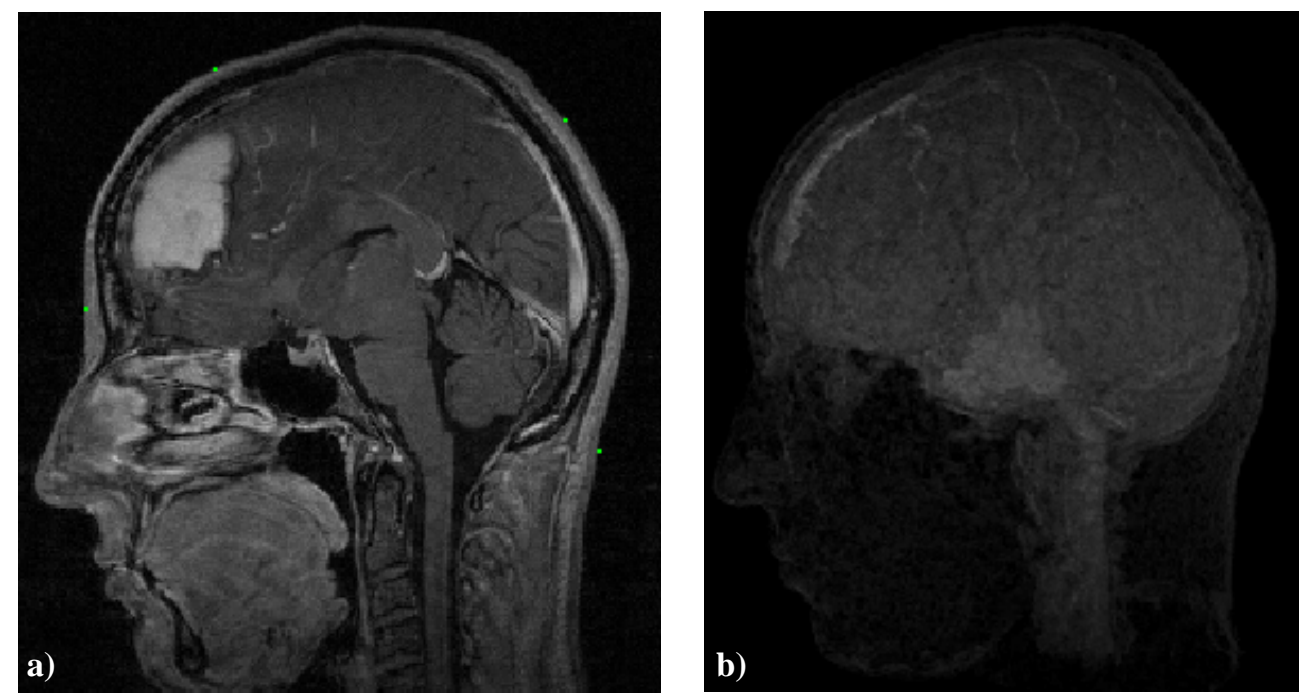

Figure 8: Skin addition: the point placement (a) and the visual result (b).

2. R. Shahidi, R. Tombropoulos, and R. P. Grzeszczuk, "Clinical applications of three-dimensional rendering of medical data sets," Proceedings of the IEEE 86, pp. 555-568, Mar. 1998.

3. I. N. Bankmann, ed., Handbook of Medical Imaging: Processing and Analysis, ch. 41-43. Academic Press, 2000 .

4. S. D. Olabarriaga and A. W. M. Smeulders, "Interaction in the segmentation of medical images: a survey," Medical Image Analysis 5, pp. 127-142, 2001.

5. S. Benini, E. Boniotti, A. Signoroni, and R. Leonardi, "Interactive segmentation of biomedical images and volumes using connected operators," in ICIP 2000, pp. III:452-455, (Vancouver, Canada), Sep. 2000.

6. R. A. Drebin, L. Carpenter, and P. Hanrahan, "Volume rendering," Computer Graphics (SIGGRAPH'88 Proc.) 22(4), pp. 65-74, 1988.

7. M. Levoy, "Volume rendering: display of surfaces from volume data," IEEE Comp. Graphics and Applications 8, pp. 29-37, May 1988.

8. B. Lichtenbelt, R. Crane, and S. Naqvi, Introduction to Volume Rendering, Prentice Hall, 1998.

9. A. Signoroni and R. Leonardi, "Volume simplification and segmentation by 3D connected operators," in EUSIPCO'02, pp. III:489-492, (Toulouse, France), Sep. 2002.

10. P. Salembier and J. Serra, "Flat zones filtering, connected operators, and filters by reconstruction," IEEE Transactions on Image Processing 4, pp. 1153-1160, August 1995.

11. G. Eckel, OpenGL Volumizer Programmer's Guide, SGI Developer Bookshelf, 1998. 